\title{
Un matemático comprometido política, social y académicamente: José Luis Massera.
}

\author{
Vernor Arguedas \\ vernor.arguedas@ucr.ac.cr \\ Universidad de Costa Rica
}

Resumen. Se analizan algunos aspectos de la vida de José Luis Massera, tanto académicos como políticos.

Palabras clave: Massera, método inverso de Lyapunov, teorema de Perron, Partido Comunista de Uruguay.

Abstract. Some aspects of the life of José Luis Massera, both academic and political are analyzed.

KeyWords: Massera, inverse Lyapunov method, Perron Theorem, Communist Party of Uruguay

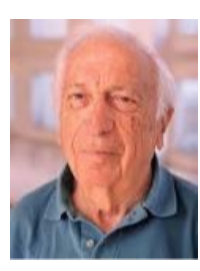

José Luis Massera (Génova, 8 de junio de 1915; Montevideo, 9 de septiembre de 2002) fue un extraordinario matemático y político comunista uruguayo. En su ámbito profesional es muy conocido por sus trabajos sobre ecuaciones diferenciales y el método inverso de estabilidad de Lyapunov.

Autor de muchos libros y artículos matemáticos, políticos y culturales, Massera conjugó el compromiso social con sus intereses académicos de una manera única.

A continuación se presenta un relato ameno, elaborado por el mismo profesor Massera, sobre su vida, en el que se mezclan nombres de matemáticos y una explicación de su trabajo que incluye un comentario de la generalización del teorema de Perron. 
Dejemos que Massera nos cuente algunos detalles de su larga carrera académica y política.

\section{"Recuerdos de mi vida académica y política}

Conferencia dictada en el Museo Nacional de Antropología, Ciudad de México - 6 de marzo de 1998 Tomado de "José Luis Massera. El científico y el hombre. Premio México de Ciencia y Tecnología" Ed: Facultad de Ingeniería, Montevideo, Uruguay (1998)

Pienso que estos recuerdos, más allá de algunos episodios con sello personal, contendrán experiencias generalizables de interés para muchos de ustedes.

Cuando cursaba el sexto año del ciclo Primario, tuve un maestro que marcó mi vida profundamente. Nada más ni nada menos, él me enseñó a pensar. Era algo adusto, no admitía fáciles simpatías, alguna vez que no olvido me sancionó, y ante la protesta de mi padre anotó en su libreta una sola palabra: "mimado", cosa que pude leer, quizás porque él quería que lo leyera. Y estuve de acuerdo con él. Lo esencial fue lo que ya dije antes: más allá de los conocimientos del programa, fue capaz de grabar fuertemente en mi mente que lo decisivo no era tal o cual aprendizaje particular, sino ayudarme a que yo mismo fuera capaz de entender, como cosa propia, mía, como pensando, hubiera podido llegar a él.

Una vez, en la clase de matemática del primer año del Liceo el tema a tratar era la semejanza de polígonos. Yo no había estudiado la lección y, justamente me llamaron para darla. Podría quizás haber inventado alguna excusa, pero acepté el reto y pasé al pizarrón donde lo que inventé fueron demostraciones de los teoremas. Honradamente no puedo asegurar que eran correctas, pero creo que no estaban descaminadas en lo esencial. No sé qué conclusiones sacaron mis compañeros de clase; de lo que sí estoy seguro es que el profesor no entendió nada: lo había sacado fuera de lo que había leído en el texto.

Más adelante, siempre en el Liceo, en general no me gustaban las materias memorísticas - nunca tuve buena memoria y ahora es mucho peor- particularmente la Historia. Hubo una excepción con un profesor no demasiado bueno pero que intentaba una explicación relativamente racional de la historia: sea como fuere, aquello me gustó. En tercer año, el profesor de matemática era un alemanote que sabía algo y me daba gusto, quizás como manifestación incipiente de mi vocación.

Más importante fue en esa época -tenía unos quince años- la revolución que comencé en mi casa y que duró varios años. Mi padre tenía un Diccionario Enciclopédico Hispano Americano en varios tomos de bastante buen nivel. Un día, de retorno de la clase del alemán, se me ocurrió buscar en el diccionario una de las palabras que había usado, probablemente "ecuación". Me encontré con una enorme cantidad de ecuaciones diferentes, que ni había sospechado que existieran, ni cómo abordarlas. Satisfecha con creces mi curiosidad con las algebraicas, fui a buscar una de las otras del diccionario. Así, día por día y palabra por palabra, comencé una recorrida, sumamente caótica, sin duda, que me fue aportando una cosecha de términos matemáticos y de informaciones valiosas sobre ellos, que iba acumulando y conceptualizando lentamente.

Por esas fechas, mis padres viajaron para un tratamiento en Carlsbad y me llevaron con mis dos hermanas. Al regreso pasamos por París, y acompañé a mi padre a una gran librería. Ahí compré dos excelentes libritos, uno sobre geometría clásica y otro de trigonometría. A la vuelta los devoré en poco tiempo: por fin encontré textos de matemática razonablemente ordenados. No obstante seguí usando el método, malo pero abundante, del diccionario.

Después del Liceo venían dos años de Preparatorios para diferentes carreras y elegí, naturalmente, Ingeniería que era la que más usaba matemática. Pero yo ya estaba más adelante del programa. Leía bien el inglés y el francés, pero conocía la importancia del alemán y contraté un curso particular intensivo de dos meses para aprender los rudimentos que necesitaba para leer matemática. Fue todo un 
éxito, practiqué -con ayuda del diccionario- leyendo un grueso volumen de Geometría Proyectiva.

El paso por Preparatorios tuvo otras consecuencias importantes. Allí conocí al colega Rafael Laguardia, que había hecho un curso superior con grandes profesores franceses. Trabamos una estrecha amistad que duró hasta su muerte en 1980. Él conocía algunos otros jóvenes aficionados a la matemática, con quienes formamos un grupo de estudio. Alguno que había leído un texto importante, daba luego un cursillo a los otros.

Pronto se abrieron nuevos horizontes. En el Uruguay todos habíamos leído buenos libros de texto del matemático español entonces radicado en la Argentina, Julio Rey Pastor, que hacía viajes anuales a Europa para actualizarse. Nos relacionamos con él, y acordamos que haría visitas los sábados a Montevideo para trasmitir a nuestro grupo esas novedades. Así se formalizó un curso bastante extenso sobre lo que entonces llamábamos "Espacios Abstractos", hoy diríamos más bien "Topología General". Posteriormente y como consecuencia de la guerra de España y de los movimientos fascistas en Europa, inmigraron a Argentina Santaló y otros españoles, el gran matemático italiano Beppo Levi y otros, que se convirtieron en nuevos profesores y amigos de nuestro grupo. Por otra parte, delegaciones nuestras concurrían a las reuniones de la Unión Matemática Argentina, que ya existía desde años atrás. Por esos mismos años, se vinculó a nuestro grupo Misha Cotlar, hijo de un modesto inmigrante ruso, que hoy es un destacado matemático a nivel mundial. En esa época, en torno al gran movimiento de solidaridad con el pueblo español, comencé mi actividad política que comparte con la matemática el resto de mi vida.

Ingresé a la Facultad de Ingeniería en 1935, año en que fue mi profesor de análisis don Eduardo García de Zúñiga, uno de los tres primeros ingenieros egresados en el Uruguay, ya muy viejo. Eligió para definir los números reales el método de las sucesiones fundamentales, quizás el más difícil de todos. Por mis intervenciones en clase supo cuál era el nivel de mis conocimientos matemáticos, lo que explica el hecho insólito de que en 1937 me designaran Ayudante de Clases Prácticas; en los exámenes subsiguientes, Zúñiga me preguntaba, para que me luciera, temas muy superiores a los que daban en los cursos. Zúñiga publicó incluso alguna investigación propia, enriqueció la Biblioteca de la Facultad, que hoy lleva su nombre, con colecciones enteras de las principales revistas matemáticas del mundo y algunas científicas muy antiguas, Obras Completas de los más grandes matemáticos, etc., todo lo cual la ha convertido en un tesoro bibliográfico probablemente único en América Latina, digno de ser consultado por historiadores de la ciencia de otros países. Ni que decir tiene, ese tesoro reunido por nuestro "gran antepasado" como lo llamábamos cariñosamente, fue un instrumento valiosísimo de nuestras investigaciones posteriores.

Cerrando este largo paréntesis, debo decir que razones reglamentarias obligaban a finalizar la carrera de Ingeniería para recibir el grado de Profesor Titular de la misma, así fuera de matemática. Así lo hice, como Ingeniero Industrial, en 1943. No fue un esfuerzo penoso, la carrera me dio una buena formación en Mecánica, Física, Química, muy útil para mi cultura científica.

Por iniciativa de Laguardia y con el apoyo inteligente del Decano Vicente García, se fundó en 1942 el Instituto de Matemática y Estadística que hoy lleva el nombre de aquél. Por fin el grupo de aficionados tenía un hogar, aunque muy modesto en su realidad material: un salón espacioso dividido por tabiques en compartimentos más pequeños. Se podía trabajar, estudiar, dar cursillos, era un cambio cualitativo.

Mientras tanto, en el mundo las cosas seguían agravándose. Derrotada la República Española, los acontecimientos se orientaban hacia la ofensiva nazi-fascista y el desencadenamiento de la Segunda Guerra Mundial. Mi actividad política consiguientemente se amplió y radicalizó. Fui designado Presidente de un Movimiento por la Paz incipiente y luego Secretario General de un gran movimiento 
de solidaridad con los aliados: Acción Antinazi de Ayuda a los Pueblos Libres, que reunía dinero, ropa y calzado de abrigo y medicamentos y los entregó a los representantes diplomáticos de la Unión Soviética, Inglaterra y Francia Libre "de acuerdo a la voluntad del donante", expresada en el momento de la donación. Terminada la guerra, y frente a los peligros de la "guerra fría", volví a la lucha por la paz, llegando a integrar el Congreso Mundial de la Paz, que dirigía el movimiento internacionalmente. En 1942 me afilié al Partido Comunista del Uruguay.

El fin de la guerra abrió perspectivas nuevas para la vida académica. La Fundación Rockefeller otorgó una beca a Laguardia para estudiar matemática en Estados Unidos. Posteriormente yo también obtuve una beca similar. Años antes, había resuelto muchos de los problemas del libro "Aufgaben und Lehrsätze der Mathematik" (Problemas y teoremas matemáticos) de dos célebres matemáticos húngaros Polya y Szegó, profesores en Stanford, y quedé muy admirado por la capacidad matemática y didáctica que mostraban en esa obra. Les escribí pues una carta manifestando mi interés en tomar algunos cursos con ellos; adjuntaba una larga lista de las obras que había estudiado hasta entonces. La respuesta de Szegó fue afirmativa y traslucía cierto asombro ante la amplitud de mis conocimientos.

Decidí entonces ir a la Universidad de Stanford en California para cumplir mi beca. En aquella época no era tan sencillo hacer el viaje en avión hasta ese lugar y pensé que lo más práctico era ir a Nueva York y desde allí usar otros medios de transporte menos caros. Inauguré así un estilo de viajes que luego apliqué en muchas ocasiones. Visité lugares y museos de Nueva York; Quebec, Montreal, Ottawa y Toronto (estupendo museo de antigüedades chinas), las Cataratas del Niágara heladas, el Cañón del Colorado, la bella San Francisco y tantos otros lugares.

En Palo Alto, un pueblito que está ubicado frente a la Universidad, era usual alojar a los estudiantes en piezas de casas particulares; así lo hice en una inmensa mansión perteneciente a uno de los propietarios de la General Motors, en que vivía su esposa de mucha edad, de la cual me hice amigo y me permitió conocer facetas peculiares de los norteamericanos. Cierta vez, me dijo que había recibido la visita de un agente de la FBI que la había interrogado sobre mí, "porque Ud. debe saber que es comunista". Ella le contestó que no tenía nada que criticarme, que mi comportamiento era correcto y que estudiaba mucho... Tratándose de una persona de esa clase social, el hecho me pareció significativo.

En Stanford me recibieron con una pequeña fiesta familiar. Con Polya tuve una relación cordial, pero no profunda. En cambio, me hice muy amigo de Szegó, su señora y su hijo Peter, al cual volví a encontrar en Nueva York como compañero de estudios, y que es hoy un destacado matemático, del cual suelo tener noticias hasta ahora. Todos ellos eran excelente gente, muy progresista. Las clases de la Universidad no eran de nivel elevado, salvo un curso de teoría de los números reales dictado, como profesor visitante, por Rademacher. Cierta vez dio una demostración muy complicada de un fino teorema de aproximación de un número real, correcta, pero que me dejó molesto: en la clase siguiente le propuse una demostración de carácter geométrico, muy breve, que lo encantó.

Paradojalmente, no fue en la Universidad sino fuera de ella que encontré un motivo de gran interés profesional. Conocí una persona que había sido Oficial de alto rango de la Marina Zarista, casado con una española muy simpática. Él trabajaba entonces para la Marina de EE.UU. en problemas de estabilización de barcos en mar agitado, y se apoyaba en estudios teóricos de matemáticos rusos sobre cuestiones de estabilidad, de los que tenía fotocopias y sabía que la Universidad de Nueva York había hecho una publicación en inglés. Conseguí un ejemplar de ésta y como en el Uruguay había adquirido algunas nociones de ruso, mal que bien pude leer también las fotocopias de mi amigo el marino. Todo lo cual fue para mí de gran interés; no así para el marino que, aunque culto, no era capaz de penetrar en el sentido matemático de las cosas. 
Estos temas me apasionaron y aunque pude cambiar ideas sobre ellos con algunos matemáticos que pasaron por la Universidad, fui convenciéndome que Stanford no podía resolverme el problema. Pedí pues a la Rockefeller traslado hacia Nueva York y Princeton, en donde se ocupaban de él, lo que me fue concedido. Viajé por lo tanto al Este y me alojé en una pieza en Nueva York. El plan de trabajo era inscribirme en las dos Universidades y viajar en ferrocarril dos o tres días por semana; los trenes, muy cómodos, permitían estudiar en el trayecto.

En la primera, dirigida por Courant - y que hoy lleva su nombre - eran profesores grandes matemáticos: Friedrichs, Artin y otros. El primer contacto me abrumó efectivamente, habían trabajado en ecuaciones diferenciales, pero habían dejado de ser un centro importante al respecto. Courant acababa de terminar un libro sobre superficies mínimas y me honró pidiéndome que revisara el texto. Era su gran "hobby" del momento, con mucha matemática fina pero también con un arsenal de alambres para definir sus contornos y una receta de agua jabonosa; estaba muy orgulloso de haber logrado una superficie mínima en forma de banda de Möbius. Trabajé mucho con él y Friedrichs en diversos temas y llegamos a ser amigos. Cuando me aprontaba para el regreso a casa, escribió una elogiosa carta al Decano de la Facultad pidiéndole su apoyo en mi carrera matemática.

El centro fundamental de mi trabajo en el Este fue, sin embargo, la Universidad de Princeton, cuyo departamento de Matemática estaba a cargo del gran matemático ruso Solomon Lefschetz. Su profesión original había sido en realidad Ingeniero Civil y trabajó como tal. Cierta vez, en Francia, dirigía una obra y, en un instante fatal zafó una linga de acero que le rebanó ambos antebrazos. Salvó la vida y le colocaron dos prótesis articuladas con dedos apoyados entre sí por resortes con los que podía sujetar algunos instrumentos elementales para escribir y otras acciones con esfuerzos corporales increíbles. Así empezó para él otra vida, profundizó el estudio de la matemática y, con un talento asombroso, se convirtió en uno de los fundadores de la Topología Algebraica moderna. En la época en que llegué a Princeton se había entusiasmado con las perspectivas promisorias que podían darse de aplicaciones de topología al estudio de las ecuaciones diferenciales, sobre lo cual había publicado un libro. Entre nosotros se gestó una profunda amistad y mutua colaboración que se prolongó hasta su muerte. Por fin, era el matemático que yo necesitaba.

En vista de esta nueva situación, pedí a la Rockefeller una extensión de la beca por seis meses más; corría el año 1948. Fue la época de mayores resultados en mis investigaciones en EE.UU.: recíproco del método de Lyapunov en la estabilidad del movimiento, descripción del conjunto de soluciones de un sistema de ecuaciones diferenciales en el plano, estudio de las soluciones periódicas armónicas y subarmónicas. En el segundo y tercero de estos temas, estudié un trabajo de un conocido matemático yanqui y me pareció encontrar un error topológico que lo invalidaba. Con cierta confusión, consulté a Lefschetz; la respuesta fue tajante: "pues si Ud. cree que hay un error, ¡dígalo!". De ahí surgió el trabajo en que corregí el error y describía exhaustivamente el conjunto infinito de las soluciones.

En el viaje de regreso a Uruguay, me detuve en las Universidades de Nueva Orleans, México y Río de Janeiro, donde expuse mis resultados, así como en el Instituto de Montevideo, donde estaban apareciendo nuevos jóvenes matemáticos: Villegas, Cabaña, Schäffer, Lumer, Sebastiani, Lewowicz. Allí Laguardia había tomado nuevas iniciativas de las cuales una importante: el Coloquio, en que con ritmo frecuente cada uno exponía los temas e investigaciones en que estaba trabajando; otros matemáticos uruguayos se doctoraban en el extranjero; el Instituto era ya conocido y apreciado en otros países y venían profesores visitantes de jerarquía (Halmos, Laurent Schwartz, etc.), a veces, países latinoamericanos nos enviaban sus jóvenes a estudiar a Montevideo.

Mientras, yo seguía estudiando y publicando nuevos resultados que completaban mis estudios de Stanford; con cierta frecuencia, Lefschetz me invitaba a concurrir a los seminarios que dirigía en la Torre de Ciencias de la UNAM, que eran muy fértiles para mí. 
En un viaje a Moscú por mis actividades político-sociales, un grupo numeroso de matemáticos rusos me invitó a la casa de uno de ellos en que me contaron que, leyendo el Annals of Mathematics, un día se sorprendieron de ver resuelto el problema inverso del método de Lyapunov, que varios de ellos habían intentado infructuosamente resolver durante años, y que estaba firmado por un matemático de Montevideo (¿dónde quedará ese país?). El viejo matemático italiano Sansone, que se ocupaba también de ecuaciones diferenciales, me invitó varias veces al Centro Matematico Estivo, que la Unione Matematica Italiana organizaba en los veranos en una villa de que disponía, a orillas del Lago de Como (¡estupendo paisaje!), donde hacían coloquios invitando a matemáticos italianos y de otros países, entre ellos Halmos, donde expuse mis resultados varios años y me enteraba de otras investigaciones en curso.

Otra actividad que desarrollé durante años fue la de colaborador en las revistas Mathematical Reviews y su par alemán: me enviaban recortes de publicaciones para que elaborara sus reseñas. En particular, como entendía razonablemente bien el ruso matemático, no era nada difícil para mí hacer las reseñas de ambas revistas en ese idioma en que no era frecuente encontrar revisores, y a la vez recibía información fresca de nuevas investigaciones. Estaba así al día en problemas de punta que alimentaban mi propia creación.

Cuando Schäffer volvió de Suiza, donde había recibido el título de Doctor, un día caminábamos conversando por el corredor del IME y yo le conté que había leído en el Mathematische Annalen de principios de siglo un pequeño trabajo de un gran matemático alemán, Perron, en que demostraba que si el segundo miembro de una ecuación diferencial lineal era acotado, existía una solución también acotada; y le comentaba que seguramente existía una vasta "generalización de ese resultado"; él estuvo de acuerdo, y resolvimos "meterle el diente" trabajando en colaboración. Mi "olfato" no me había engañado, y relativamente pronto publicamos en la misma revista varios trabajos con generalizaciones sucesivas. Resolvimos entonces "agarrar el toro por los cuernos", y emprender una amplísima generalización del teorema de Perron. La variable independiente de las ecuaciones seguía siendo la variable real $t$, pero la variable dependiente pasaba a ser ella misma una función medible en un espacio de Banach X, o sea, la variable dependiente se "movía" en un espacio funcional.

Para que resulten claros, al menos para algunos de Uds. los conceptos siguientes, me veo obligado a introducir algunos tecnicismos matemáticos aunque no totalmente precisos. Consideraremos dos tipos de ecuaciones diferenciales lineales, homogéneas

$$
\frac{d x}{d t}+A y=0
$$

y no homogéneas

$$
\frac{d x}{d t}+A x=f(x)
$$

en que $A$ es un operador lineal que actúa sobre $X$ y $f$ una función medible sobre $X$. Diremos que el subespacio $Y$ de $X$ induce una "dicotomía" de las soluciones de 1.1 si cuando las condiciones iniciales de una solución pertenecen a $Y$, la solución es estable, e inestable en caso contrario. Diremos que la pareja $(B, D)$ - donde $B$ y $D$ son dos espacios funcionales en $X$ - es "admisible" para 1.2 si para cada $f$ perteneciente a $B$ existe una solución de 1.2 perteneciente a $D$ (recuérdese el teorema de Perron). Los resultados principales de la investigación pueden entonces sintetizarse en el siguiente teorema: "Si existe una pareja admisible para la ecuación no homogénea 1.2, entonces la ecuación homogénea 1.1 posee una dicotomía, y recíprocamente". Los resultados de esta investigación fueron publicados en un libro de 400 páginas editado en Estados Unidos que fue traducido al ruso. Posteriormente, fui invitado a exponerlos en un seminario internacional acerca de los espacios funcionales que se celebró 
en Israel.

En 1955, el Partido Comunista del Uruguay atravesó una grave crisis: su Secretario General fue expulsado por el Comité Central (C.C.) que yo integraba, y reemplazado por Rodney Arismendi, viejo amigo mío, cuyo informe fue removedor. Se abría paso la idea de que la revolución uruguaya no podía aún ser socialista. Un primer paso en ese camino de una "Democracia Avanzada" fue en 1962 el Frente Izquierda de Liberación (FIDEL) presidido por Luis Pedro Bonavita, pequeño productor rural, ex - integrante de uno de los partidos burgueses.

En aquella reunión del Comité Central a la que aludí pasé a ser miembro del Comité Ejecutivo del Partido Comunista. En 1963-1972 fui electo diputado nacional en listas del FIDEL. La tarea parlamentaria, muy absorbente, me impedía toda actividad académica, salvo el simple dictado de clases en la Facultad. La situación política en el país empezó a tensarse, hubo violentas represiones, estudiantes asesinados en las calles; la Universidad, de la cual era Rector mi colega y amigo Oscar Maggiolo, fue duramente hostigada.

Al mismo tiempo, maduró en 1971 la creación de una nueva alianza política, el Frente Amplio (FA), hoy día tercera fuerza política nacional y con la posibilidad de disputar en las elecciones de 1999 la presidencia de la República a los dos partidos tradicionales. Está compuesta por socialistas, comunistas, fidelistas, democristianos, anarquistas y otros grupos políticos de izquierda, y algunos nuevos desprendimientos de los partidos burgueses tradicionales "blanco" y "colorado". Para presidirlo se eligió al General (r) Líber Seregni y con él llegaron al Frente Amplio decenas de militares demócratas.

Se preparaba el golpe militar de derecha de junio de 1973. En la Facultad de Ingeniería, donde era Decano otro gran amigo, el ingeniero Julio Ricaldoni, hubo un accidente explosivo que se atribuyó a estudiantes de izquierda, la Universidad fue intervenida, casi todos los Decanos fueron presos, entre ellos Ricaldoni, por varios años.. Muchos destacados intelectuales y militantes fueron asesinados; otros pudieron exiliarse en países de América y Europa. La embajada de México fue el hogar de muchos refugiados que luego vivieron muchos años entre ustedes y profesan a México un amor entrañable.

La mayoría de los matemáticos se exiliaron. Yo pasé a la clandestinidad unos pocos años. Lewowicz antes de exiliarse - me dio refugio en su casa varias noches. Un tiempo ejercí la secretaría del P.C.U. en el territorio nacional. Mi hijo, que era muy enfermo, murió a principios del 73; mi hija se exilió con su familia en Brasil, donde vivieron hasta el fin de la dictadura. La hija de Martha que quiero como si fuera mía, se exilió en México con sus dos hijitos.

Caí preso en octubre de 1975; el primer día, estando de plantón y con manos y tobillos atados, un soldado me tomó de los hombros y me desplazó bruscamente, caí y me fracturé el cuello del fémur derecho, no obstante lo cual siguieron los plantones hasta que se convencieron de que era imposible que me mantuviera en pie y me acostaron en un jergón de alambre donde estuve un mes sin asistencia hasta que un médico indicó que me llevaran al hospital militar para hacer una radiografía. A partir de ahí, con la ayuda de un bastón rudimentario, el organismo mismo se encargó de soldar la fractura, hasta hoy.

Permanecí preso nueve años y medio en dos cuarteles y una prisión conocida como Penal de Libertad; el nombre sarcástico se debe a que está ubicado en las cercanías de un pueblo con ese nombre. Las celdas del Penal alojaban dos presos, con mínimas posibilidades de comunicación con los restantes, salvo en algunas tareas colectivas de la vida diaria, en los llamados "recreos" de una hora en que los que podían hacían deportes y los que no, como era mi caso, caminábamos conversando con otro preso; y las visitas familiares cada quince días, también de una hora de duración. Como puede verse, 
las relaciones humanas estaban limitadas casi exclusivamente a la celda.

En ella podía leerse libros de la buena biblioteca que se había ido formando con las donaciones que hacían los familiares de los presos cuando se lo permitían. Eso sí, con exclusión de libros políticos... y de matemática. ¡Vaya a saber que misteriosos mensajes podían contener aquellos signos raros e incomprensibles!. Hubo alguna falla en esa disposición y me tocó a mí, precisamente. Una vez, pedí a la biblioteca un libro cuyo titulo era confuso; el libro llegó, no tenía tapas sino una cubierta "casera" hecha con papel grueso. Empecé a leerlo y su estilo me resultaba de alguna manera conocido; efectivamente, pocas páginas después el misterio se aclaró en una nota al pie: era una edición soviética traducida al español de un texto del famoso matemático inglés Turing en que explicaba el "mecanismo" de la llamada máquina que lleva su nombre y que tanta importancia teórica tiene para ramas de la matemática moderna; lo estudié concienzudamente, con una sonrisa pícara que no abandonaba mis labios... Años después, una versión teatralizada de la vida de Turing, excelente, se representó en el Uruguay durante varios años. La biblioteca del Penal contenía una gran riqueza de obras literarias, entre ellas mexicanas, que leí durante esos largos años. Recuerdo especialmente "La muerte de Artemio Cruz" de Fuentes.

Los compañeros de celda fueron varios, comunistas y tupamaros, con los que se conversaba libremente de los más variados temas. Un obrero de una fábrica de papel, comunista, me acompañó durante años y nos hicimos grandes amigos; muy inteligente e inquieto, hablamos de los más diversos temas; incluso, recordando enseñanzas de la Facultad, pude darle cursillos de física, química, etc., que absorbía con pasión. En otras celdas estuvieron presos jóvenes matemáticos como Markarian y Accinelli, a los que veía sólo en los recreos y en alguna tarea colectiva; corriendo algunos riesgos, hicimos algunos trabajitos matemáticos como el titulado "¿Es cierto que dos más dos son siempre cuatro?", que podían interesar e intrigar a presos no matemáticos.

A todo esto, Martha, mi esposa, también había caído presa, fue torturada y recluida en una cárcel de mujeres, instalada en lo que había sido un monasterio. Estuvo allí tres años hasta avanzado el año 1979; pudo recuperar nuestro apartamento, que había sido ocupado y saqueado por los militares. Logró vivir dando clases particulares de filosofía y venia a visitarme al Penal venciendo las infinitas molestias y requisitos interpuestos. Mientras estuvo presa nos carteamos esporádicamente porque no siempre nos entregaban nuestras cartas. Entonces, me visitaba y atendía mi hermana menor.

El exilio en otros países americanos y europeos fue el destino, también difícil, otros muchos compatriotas. En el caso de los matemáticos y otros científicos profesionales, les fue más fácil que se reconociera sus capacidades. Para muchos otros obtener empleos y trabajo costó mucho. Una de sus tareas principales fue desarrollar el movimiento de solidaridad y lucha contra la dictadura militar uruguaya. En particular debo expresar mi profundo agradecimiento por el amplísimo vuelo que tuvo, en América y Europa, el reclamo de mi libertad; aquí en México la actividad y la fuerza de solidaridad fue notable, en particular entre los matemáticos. Esa solidaridad tuvo una forma peculiar de expresión en las más de diez Universidades - entre ellas la de Puebla - que me concedieron el título de Doctor Honoris Causa.

La solidaridad internacional y la lucha democrática del propio pueblo uruguayo estaban corroyendo las bases de la dictadura. A fines de 1980 los propios militares convocaron a un plebiscito para reformar la Constitución al gusto de ellos, y perdieron. A fines de 1983, todos los partidos democráticos y las fuerzas sociales convocaron una demostración amplísima contra el régimen presidida por un estrado con notorias figuras de todos ellos, entre los que estuvo mi esposa. El público fue probablemente más grande que se haya visto en Montevideo: el lugar era un gran parque y la fotografía que se tomó desde lo alto muestra una multitud compacta que se desplegaba en curva sinuosa de una ancha avenida. Esas fotos fueron publicadas por el diario conservador "El País" que se recibía en la 
biblioteca del Penal; los compañeros que trabajaban allí difundieron el acontecimiento a todos los presos, incluido el color rojo del vestido de Martha.

El 3 de marzo de 1984 fui puesto en libertad. A partir de entonces y durante meses, mi casa fue invadida por centenares de amigos que vinieron a saludarme. También en marzo liberaron a Seregni, con quien nos encontramos en su casa, en medio de una gran manifestación callejera espontánea. Ese día había llegado a Montevideo una delegación italiana solidaria conmigo, uno de sus integrantes comentó que aquello le recordaba la caída del fascismo en su país.

Participé con Martha en muchas manifestaciones públicas que reclamaban liberación de los que continuaban presos, con emocionantes reencuentros con viejos amigas y amigos, unos que habían estado presos y otros opositores activos a la dictadura Los frenteamplistas participamos en la llamada Concertación Programática, en que representantes de los partidos políticos democráticos elaboraron una propuesta avanzada del nuevo gobierno; lamentablemente esa forma de democracia, casi directa, fue mínimamente aplicada por el gobierno democráticamente electo en 1985.

En aquel mismo año, asistimos conmovidos con el ex-Decano Ricaldoni -que había sido liberado antes que yo- y con otros docentes, a una asamblea de estudiantes en la Facultad de Arquitectura: como ellos no habían vivido en una Universidad democrática, no les resultaba fácil entender los criterios de la participación democrática en su dirección. En el verano 1984 - 85 también me emocioné en una reunión con matemáticos y otros científicos uruguayos, bajo el escudo de la UNESCO que lo auspiciaba, venidos a Montevideo desde el exilio por pocos días para discutir el Programa de Desarrollo de las Ciencias Básicas (PEDECIBA). El objetivo era reconstruir la ciencia en el Uruguay y promover el retorno de los que estaban fuera. Personalmente, fue el reencuentro con colegas, discípulos y amigos y la reanudación del trabajo académico. Fue electo como su presidente el biólogo uruguayo de fama mundial Dr. Caldeyro-Barcia; yo formé parte de la primera Comisión Directiva. El PEDECIBA es actualmente financiado por el Estado y por las Naciones Unidas (PNUD) y es una pieza muy importante del equipo de investigación científica del Uruguay.

Con el retorno democrático a la Universidad en 1985, fuimos electos el Ing. Luis Abete como Decano de la Facultad, el excelente compañero Ing. Agustín Cisa y yo como miembros del Consejo de nuestra casa de estudios. La rectificación de la conducta universitaria impuesta por la dictadura no fue nada sencilla y encontraba muchos obstáculos de otros miembros provenientes del período anterior; el clima reinante no era todavía el más adecuado. Propuse entonces hacer entrevistas con cada uno de estos "profesores" para acordar con ellos los libros relativamente interesantes que cada uno debía estudiar para estar en condiciones de dictar clases, y así se hizo. El resultado fue brillante: casi todos renunciaron y se los sustituyó por otros mejores. Simultáneamente, trabajamos con jóvenes estudiantes bien dotados que hoy son matemáticos e investigadores y recibieron su doctorado en Brasil: Markarian, los hermanos Paternain, Catsigeras, Enrich, Accinelli y otros, sin contar con los repatriados del exilio.

Después de tan duras pruebas, hoy la escuela matemática uruguaya es fuerte. En el Instituto de Matemática y Estadística Prof. Ing. Rafael Laguardia (IMERL) se estudian Sistemas Dinámicos y Caos. En otra sede importante, el Centro de Matemática de la Facultad de Ciencias, se estudian otras ramas. Paralelamente, he integrado (primero con Ricaldoni y Rafael Guarga, actual Decano, y luego con María Simon y Arturo Lezama) la Comisión de Investigación Científica de la Facultad de Ingeniería.

Ya en 1973, había previsto que sería imposible reanudar mis investigaciones matemáticas luego de la dictadura habiendo pasado casi treinta años sin hacerlo. Me gustaba mucho la historia de la matemática y de la ciencia en general y tenía gran interés en hacerme una pequeña biblioteca sobre esos temas. Sólo mucho después pude emprender ese proyecto y los resultados no fueron numerosos, 
aunque de cierto interés. Un conocido filósofo francés me invitó a colaborar en un libro que él dirige, sobre la ciencia y la dialéctica, en el que escriben distinguidos científicos de diversas ramas. Acepté la propuesta; se espera que el libro aparezca este año. El contacto con Séve me ha enriquecido mucho en temas filosóficos. Termino. Pido excusas por la extensión de esta charla y espero que ella les haya sido útil como experiencia de la formación de una escuela, en este caso la Escuela Matemática del Uruguay. "

La obra matemática de Massera es muy extensa.

En [1] se encuentra su famosa obra Linear differential equations and functions spaces, escrita conjuntamente con Juan Schaffer, "Linear differential equations and function spaces"

Las publicaciones citadas en su autobiografía se pueden consultar en [2, 3].

Massera representa la importancia del compromiso social y político en el quehacer académico.

\section{Bibliografía}

[1] Massera, J. L. \& Schäffer, J. J. (1966). Linear differential equations and function spaces. New York \& London: Academic Press. http : //bookzz . org/md5/17edaf7fbb90e03633ccalb79e78091c

[2] Massera, José Luis (1949), "On Liapounoff's conditions of stability", Annals of Mathematics. Second Series (The Annals of Mathematics, Vol. 50, No. 3) 50 (3): 705-721.

[3] Massera, José Luis (1950), "The existence of periodic solutions of systems of differential equations", Duke Mathematical Journal 17 (4): 457-475

Un matemático comprometido política, social y académicamente: José Luis Massera. . Vernor Arguedas Derechos Reservados (C) 2015 Revista digital Matemática, Educación e Internet (http://tecdigital.tec.ac.cr/revistamatematica/) 